\title{
Construction of ecological security pattern based on the importance of ecosystem service functions and ecological sensitivity assessment: a case study in Fengxian County of Jiangsu Province, China
}

\author{
Xingxing $\operatorname{Jin}^{1} \cdot$ Luyao Wei $^{1}$ (D) $\cdot$ Yi Wang $^{2} \cdot$ Yuqi Lu ${ }^{1,3,4}$
}

Received: 11 October 2019 / Accepted: 7 January 2020 / Published online: 13 January 2020

(C) The Author(s) 2020

\begin{abstract}
The construction of ecological security pattern is one of the important ways to alleviate the contradiction between economic development and ecological protection, as well as the important contents of ecological civilization construction. How to scientifically construct the ecological security pattern of small-scale counties, and achieve sustainable economic development based on ecological environment protection, it has become an important proposition in regulating the ecological process effectively. Taking Fengxian County of China as an example, this paper selected the importance of ecosystem service functions and ecological sensitivity to evaluate the ecological importance and identify ecological sources. Furthermore, we constructed the ecological resistance surface by various landscape assignments and nighttime lighting modifications. Through a minimum cumulative resistance model, we obtained ecological corridors and finally constructed the ecological security pattern comprehensively combining with ecological resistance surface construction. Accordingly, we further clarified the specific control measures for ecological security barriers and regional functional zoning. This case study shows that the ecological security pattern is composed of ecological sources and corridors, where the former plays an important security role, and the latter ensures the continuity of ecological functions. In terms of the spatial layout, the ecological security barriers built based on ecological security pattern and regional zoning functions are away from the urban core development area. As for the spatial distribution, ecological sources of Fengxian County are mainly located in the central and southwestern areas, which is highly coincident with the main rivers and underground drinking water source area. Moreover, key corridors and main corridors with length of approximately $115.71 \mathrm{~km}$ and $26.22 \mathrm{~km}$, respectively, formed ecological corridors of Fengxian County. They are concentrated in the western and southwestern regions of the county which is far away from the built-up areas with strong human disturbance. The results will provide scientific evidence for important ecological land protection and ecological space control at a small scale in underdeveloped and plain counties. In addition, it will enrich the theoretical framework and methodological system of ecological security pattern construction. To some extent, it also makes a reference for improving the regional
\end{abstract}

Xingxing Jin and Luyao Wei contributed equally to the work.

Extended author information available on the last page of the article 
ecological environment carrying capacities and optimizing the ecological spatial structure in such kinds of underdeveloped small-scale counties.

Keywords Ecological importance $\cdot$ Ecological sensitivity $\cdot$ Ecological sources $\cdot$ Ecological corridors $\cdot$ Ecological security pattern $\cdot$ Fengxian County of Jiangsu Province China

\section{Introduction}

At present, with the acceleration of industrialization and urbanization, the phenomena of population growth and urban expansion appear to be more and more prominent, which have exerted tremendous pressure on resources and the environment. The ecological environment has reached a critical state, which is accompanied by a series of problems such as loss of biodiversity, aggravation of soil and water losses and land desertification (Fang et al. 2019; Plieninger et al. 2015; Yao et al. 2019). The problem has threatened the safety of human life and property as well as the sustainable development of society and the economy seriously. There is an urgent need to control and improve the ecological environment from the source, as well as to handle the relationship between economic development and ecological protection correctly. The ecological security pattern has become one of the important ways to alleviate the contradiction between economic development and ecological protection. It also plays an active role in ecological civilization construction and territorial spatial pattern formation (Cumming and Allen 2017; Liu 2016).

In recent years, relevant scholars have effectively explored the ecological security pattern construction of prefecture-level cities and urban agglomerations based on different scales (Chen et al. 2018; Wang and Xu 2018; Wang et al. 2019). Among the existing studies, it has formed a mainstream research paradigm to construct regional ecological security pattern based on the identification of ecological sources and ecological corridors, and it has been widely applied in relevant practices (Klar et al. 2012). It is noteworthy that two main methods are used to identify the ecological sources including the qualitative evaluation of ecosystem structure and the comprehensive evaluation of quantitative indicators (Peng et al. 2017). For instance, according to the natural background conditions and the characteristics of habitat patches of the study area, relative researchers regarded nature reserves, forest parks, wetland parks and other important ecological lands as ecological sources directly (Tang et al. 2011; Yu 1996), while to a large extent, the qualitative identification of ecological sources ignored the internal differences. Relevant scholars tried to identify the ecological sources quantitatively by constructing comprehensive evaluation indicators in order to avoid this problem (Gurrutxaga et al. 2010). On the whole, it could be divided into two major venations. On the one hand, some researchers identified ecological sources by analyzing regional typical ecosystem service functions and their differentiation law from a single perspective (Peng et al. 2018). However, owing to the dynamism of ecosystem service functions, it would affect the identification results to some extent when simply considering various types of services as equally important. Therefore, some scholars tried to identify ecological sources based on ecosystem service value reconstruction (Frelichova et al. 2014; Scholte et al. 2015). On the other hand, some researchers identified ecological sources based on a composite perspective (Chen et al. 2008; Li et al. 2019). The identification and functional division of ecological sources will lead to the sustainable development of the ecosystem and relevant ecological policies formulation. Taking into account the ecosystem's own functions and the response of human activities to the ecosystem, they further 
determined the ecological sources by comprehensive evaluation of ecosystem service functions importance and ecosystem sensitivity (Guo et al. 2007; Kareiva et al. 2007; Li et al. 2011). It not only concerns the quantitative evaluation of ecological patterns, but more importantly, this method also gives attention to the evolution of the ecological process.

While identifying the ecological corridors, the minimum cumulative resistance (MCR) model based on the GIS platform had been widely used (Knaapen et al. 1992; Yu 1996, 1999). At present, the construction of resistance surfaces is generally based on related coefficients such as land cover type and topographical features (Fu et al. 2010). It requires to further consider the spatial differences caused by various ecological problems among different places. The MCR model is able to simulate the landscape obstacles to spatial motion processes in a more intuitive way (Song and Qin 2016). The key to the application of this model is the construction of ecological resistance surface. Currently, the existing resistance surface construction was mostly based on the assignment operations of different landscapes (Yang et al. 2018). Although it reflected the interaction between landscape patterns and ecological processes to a certain extent, it could not reveal the inherent complexity of human activities and ecological processes. Besides, it also tended to be too subjective. The areas with relatively high human activity intensity show a certain effect on the migration of species (Zhang et al. 2017a). Night lighting data are actually a comprehensive representation of the intensity of human spatial activity (Mellander et al. 2015). Therefore, some scholars tried to modify the resistance surface value by nighttime lighting (Elvidge et al. 2009), impervious surface index (Wang et al. 2018) and other data to obtain a more scientific ecological resistance surface. Although scholars have different methods of identifying ecological sources and ecological corridors, they are all useful explorations of theories and methods for constructing ecological security patterns.

At present, relevant studies mostly evaluated the regional ecological security pattern from the single ecosystem service functions importance perspective or the composite perspective combining with ecological sensitivity. In general, they lacked comprehensive consideration of the impact of different types of landscapes. Meanwhile, they also ignored the impact of human activities on the ecological resistance coefficient to some extent. Less consideration of the subjective impact on the resistance coefficient assignments and lack of reasonable revise led to a too subjective and non-operational scheme for ecological security pattern. In addition, relevant studies mostly concentrated on the identification of ecological sources in economically developed areas such as prefecture-level cities or urban agglomerations. As for the areas with the underdeveloped economy and huge contradiction between economic development and ecological protection, there is a lack of in-depth practical discussion on ecological security pattern construction. As for developing countries, it is an important proposition in the process of urbanization to explore the optimization model of territory spatial structure by constructing an ecological security pattern (Xin et al. 2016). Meanwhile, compared to mountainous and hilly areas that obtained income from diverse landscapes and tourism development, small-scale counties in plain areas require to further solve the contradiction between economic development and ecosystem protection urgently, while the construction method of the ecological security pattern in small-scale counties of plain areas was still unclear currently. Accordingly, we took Fengxian County in Huanghuaihai Region Plain of China as a study area to discuss the methodology to identify the ecological sources and ecological corridors. Furthermore, we constructed the ecological security pattern and finally obtained an ecological security barrier combining with regional functional zoning. According to the ultimate complex and multiple ecological spatial structure system, we proposed district governance recommendations and countermeasures of ecological space so as to coordinate the spatial relations and perfect the spatial 
functions. In general, this study provides a new research framework for identifying the ecological security pattern of small-scale counties in underdeveloped plain areas. It also provides policy guidance for spatial optimization in restricted development areas where there is a contradiction between economic development and ecological protection.

The specific construction methodology of ecological security pattern is as follows: First, we obtained ecological importance evaluation result to further identify the ecological sources based on ecosystem service functions and ecological sensitivities. Next, we adopted nighttime lighting data to modify the ecological resistance surface assigned by land use classifications. Then, according to the identification of ecological sources and the construction of ecological resistance surface, we used the minimum cumulative resistance model to identify the ecological corridors. Finally, the ecological security pattern was comprehensively constructed. By further clarifying the specific measures of ecological safety barriers construction and spatial function management, we attempted to provide decisionmaking suggestions for the sustainable development of county units in the plain area and the optimal layout of territory space. The methodology framework of ecological security pattern construction is shown in Fig. 1.

The following section describes the study area and data sources. And the third section presents analytical methods that include ecological importance evaluation and ecological corridors identification. The section on findings demonstrates the analysis results. Combining with the assessment of ecosystem service functions importance and ecological sensitivity, we identify ecological sources and then construct an ecological safety pattern. The conclusion and discussion parts offer a summary of the findings, current research deficiencies and future research prospects.

\section{Study area and data sources}

\subsection{Study area}

Fengxian County is located in the northwestern part of Jiangsu Province, China $\left(116^{\circ} 21^{\prime} 15^{\prime \prime}-116^{\circ} 52^{\prime} 03^{\prime \prime} \mathrm{E}, 34^{\circ} 24^{\prime} 25^{\prime \prime}-34^{\circ} 56^{\prime} 27^{\prime \prime} \mathrm{N}\right)$. As a junction of seven counties in four provinces of Jiangsu, Shandong, Henan and Anhui and the central zone of Huaihai Economic Zone, it plays an important role. It presents an obvious geographical location advantage with an area of $1450.2 \mathrm{~km}^{2}$ and 15 townships (Fig. 2). The current situation of Fengxian's ecological security will not only affect its own sustainable development, but also have a great enlightening effect on the ecological environment protection in some areas of adjacent Jiangsu, Shandong, Henan and Anhui Province. As can be seen from the land use classifications in Fengxian County, artificial and semi-artificial ecosystems occupy a large account. The areas of cultivated land, garden land and residential land account for $84.55 \%$ of the total area, while forest land, grassland and water area account for only $5.70 \%$ of the whole county. Human activities have a certain impact on the composition of the ecosystem.

Owning sufficient light and water conditions, Fengxian County has become an important grain and fruit producing county in Jiangsu Province, China, as well as one of the main agricultural production areas at the provincial level. According to the major function-oriented zoning of Jiangsu Province issued by the People's Government of Jiangsu Province, Fengxian County is listed as a limited development zone, which is in urgent to control and solve ecological problems from the source effectively. In order to maintain 


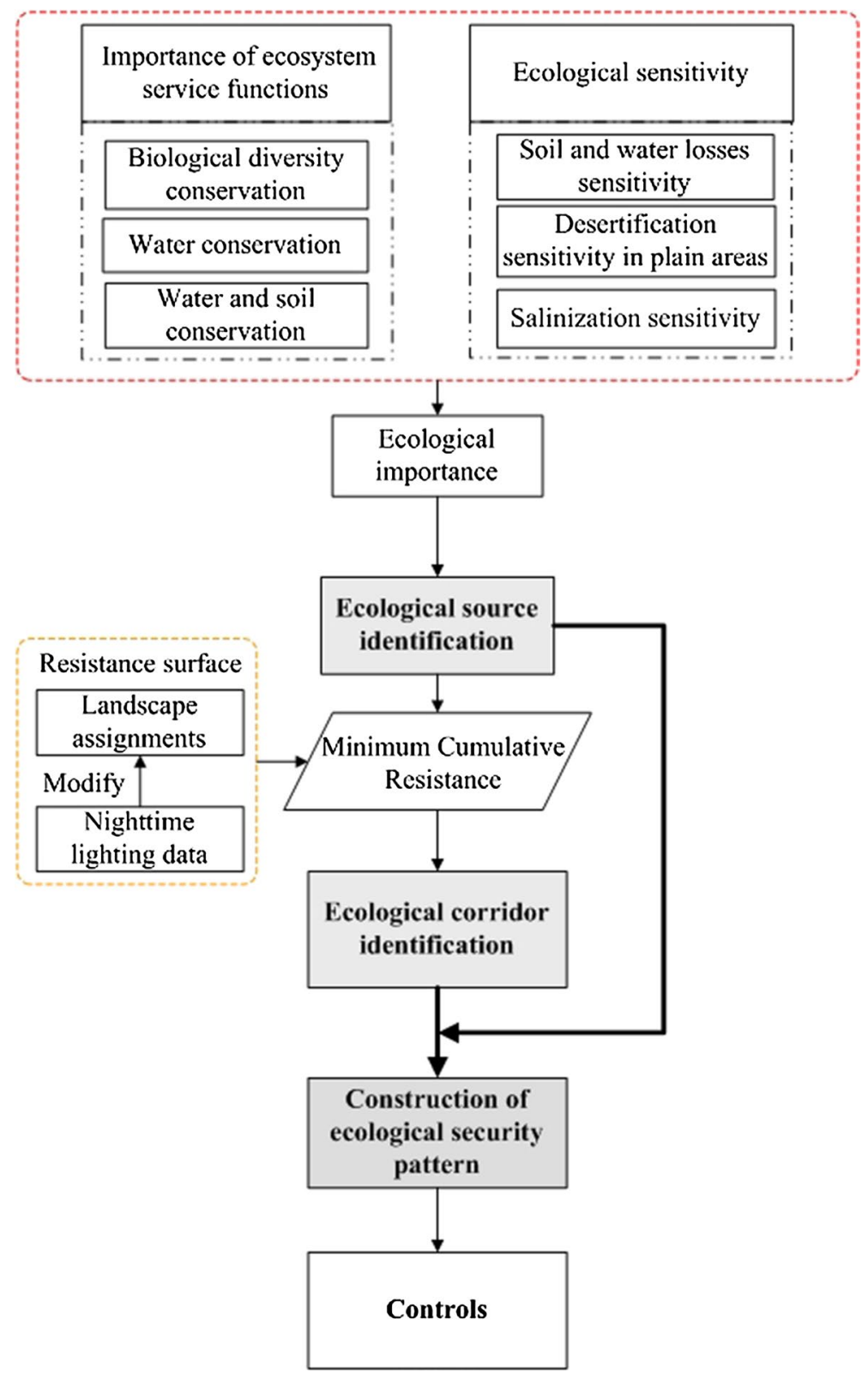

Fig. 1 The methodology framework of ecological security pattern construction

and improve the production capacity of agricultural products, it is of great need to restrict the large-scale and high-intensity industrialization and urbanization development activities in Fengxian County. A series of ecological and environmental problems have arisen due to the fast development of townships and agriculture, such as loss of biodiversity, aggravation of soil and water losses and land desertification. Moreover, 


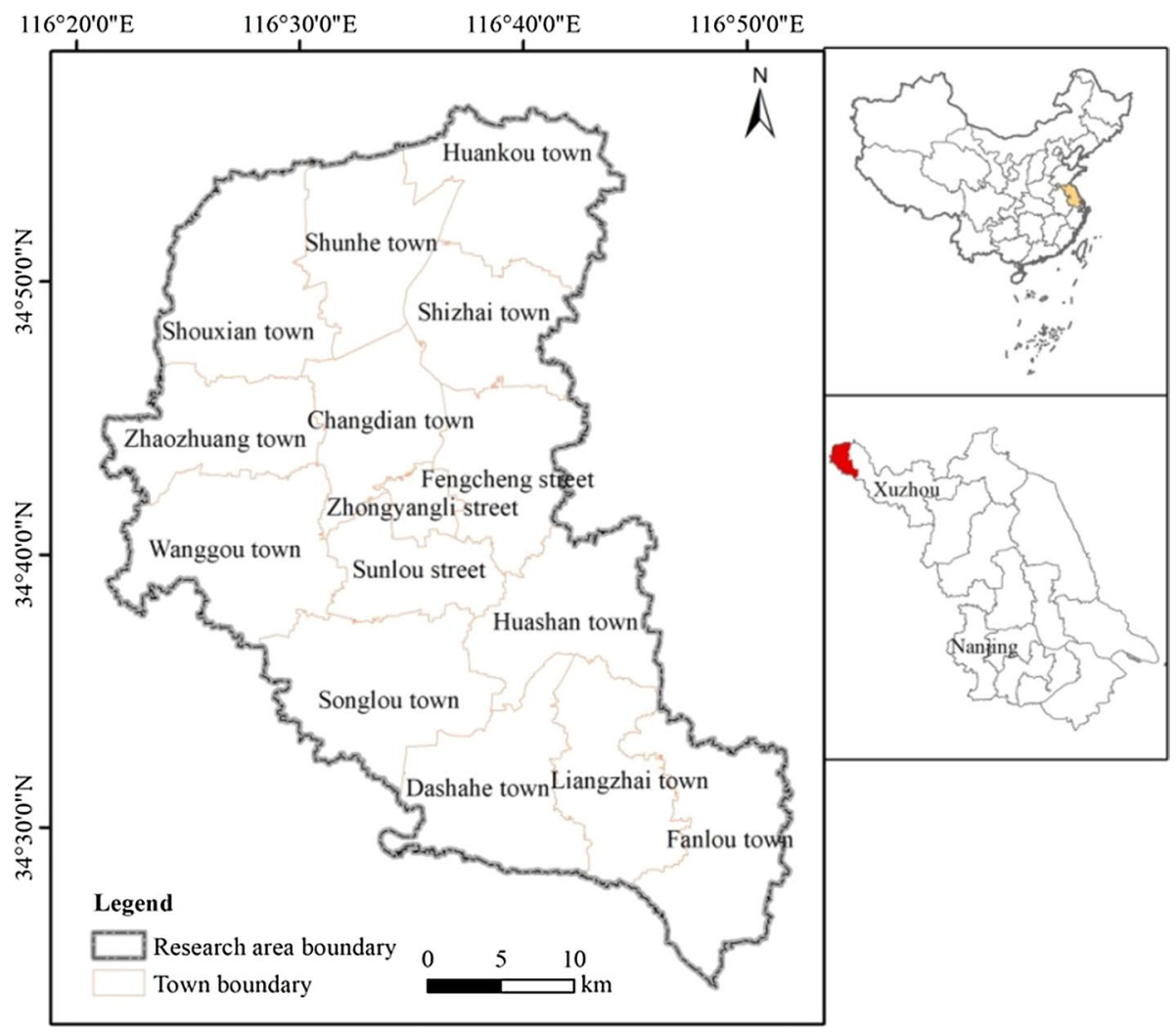

Fig. 2 The location of the study area

the contradiction between development and protection has become increasingly prominent. As a typical county unit in the underdeveloped plain area, it is conducive to alleviate the contradiction between economic development and ecosystem protection when exploring the construction mode of ecological security network patterns. It will also provide a scientific reference for the realization of regional coordinated and sustainable development. By establishing a scientific and rational ecological security pattern, we will achieve the overall planning and comprehensive regulation of territory space and coordinate the relationship between social-economic development and ecological environmental protection from the origin.

\subsection{Data sources}

The data used in this paper mainly included six types as shown in Table 1: net primary productivity (NPP), meteorological data, land use data, digital elevation model (DEM), normalized differential vegetation index (NDVI) and soil texture data (Xu 2018). To ensure the accuracy of data, the projection coordinate system was unified as GCS Xian 1980 , and all data were converted into raster images with a resolution of $30 \mathrm{~m} \times 30 \mathrm{~m}$ (Table 1). 


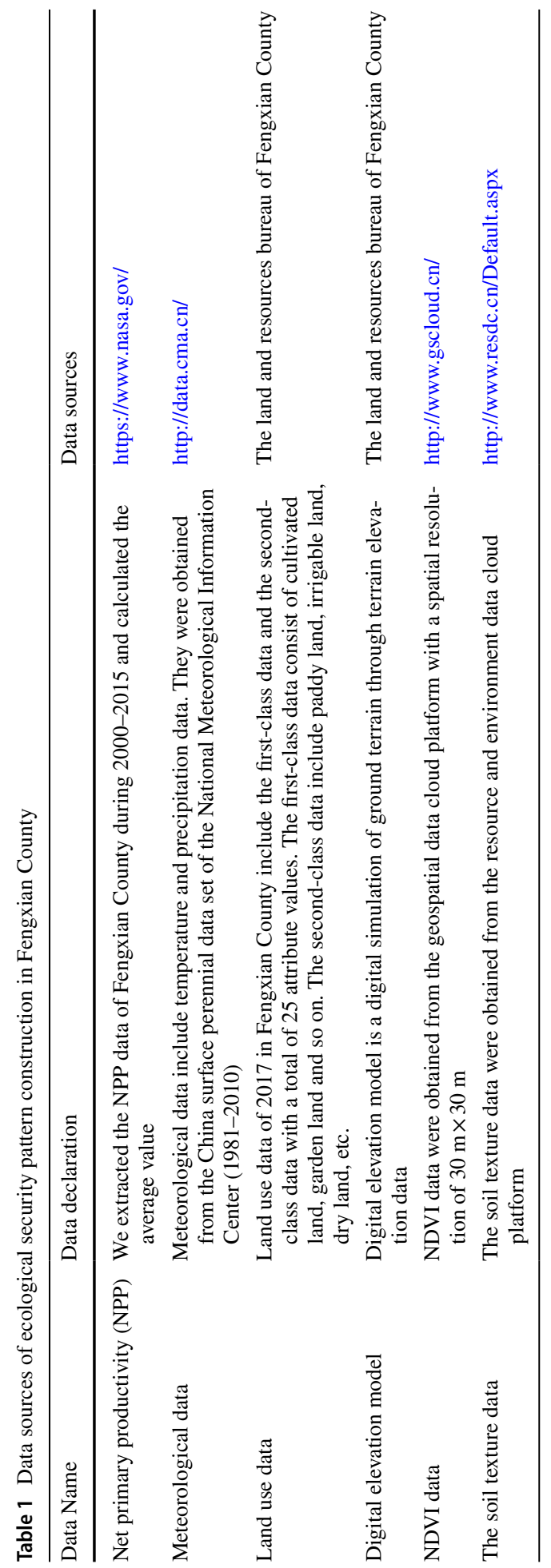




\section{Research methods}

\subsection{Ecological importance evaluation}

The ecological importance assessment refers to the comprehensive effects of the quality of service functions provided by the ecosystem and the sensitivity to external disturbances of the ecosystem. On this basis, we regard the patches with high ecological value as alternative areas of ecological sources.

\subsubsection{Importance of ecosystem service functions}

Ecosystem service functions refer to the environmental conditions and beneficial effects through which ecosystems and species sustain and fulfill humanity's survival (Daily 1997), including water resource conservation, soil and water conservation, biodiversity conservation, etc. It can identify areas with important ecological functions and provide scientific evidence effectively for ecosystem restoration and ecological function zoning by carrying out researches on ecosystem service functions importance evaluation. At present, the methods for ecosystem service functions importance evaluation are approximately divided into the model evaluation and NPP quantitative indicators evaluation (Barral and Maceira 2012; Carreño et al. 2012). According to the actual characteristics of Fengxian County and the data acquisition situation, we adopted model evaluation and NPP quantitative indicators evaluation comprehensively to evaluate the importance of ecosystem service functions (Table 2). According to the evaluation results of the importance of water resource conservation, soil and water conservation and the biodiversity conservation function, we graded the sub-dimension indexes separately. After weighted calculation, we finally got the importance evaluation of ecosystem service functions.

3.1.1.1 Importance of water resource conservation The water resource conservation is closely related to surface coverage, precipitation and terrain conditions (Cheng and Shi 2004). The water resource conservation mainly refers that the ecosystem gives full play to flood detention, peak discharge reduction, water regulation, soil and water losses reduction, etc. by intercepting rainwater through vegetation, reducing surface runoff and slowing down its velocity. The formula is as follows:

$$
\mathrm{WRC}=\sum_{i}^{j}\left(P_{i}-R_{i}-\mathrm{ET}_{i}\right) \times A_{i} \times 10^{3}
$$

where WRC denotes the importance of water resource conservation, $P_{i}$ is the precipitation $(\mathrm{mm}) ; R_{i}$ is the surface runoff $(\mathrm{mm}) ; \mathrm{ET}_{i}$ is the evapotranspiration $(\mathrm{mm}) ; A_{i}$ is the area of ecosystem type $i\left(\mathrm{~km}^{2}\right)$; and $j$ is the number of ecosystem types in the study area. The formula for $R_{i}$ is:

$$
R_{i}=P_{i} \times \alpha
$$

where $\alpha$ is the average surface runoff coefficient (Table 3), and the specific assignments of ecosystem types are referred to the policy document "Technical Guidelines for Assessment of Resources and Environmental Carrying Capacity and Suitability of Territory Space 


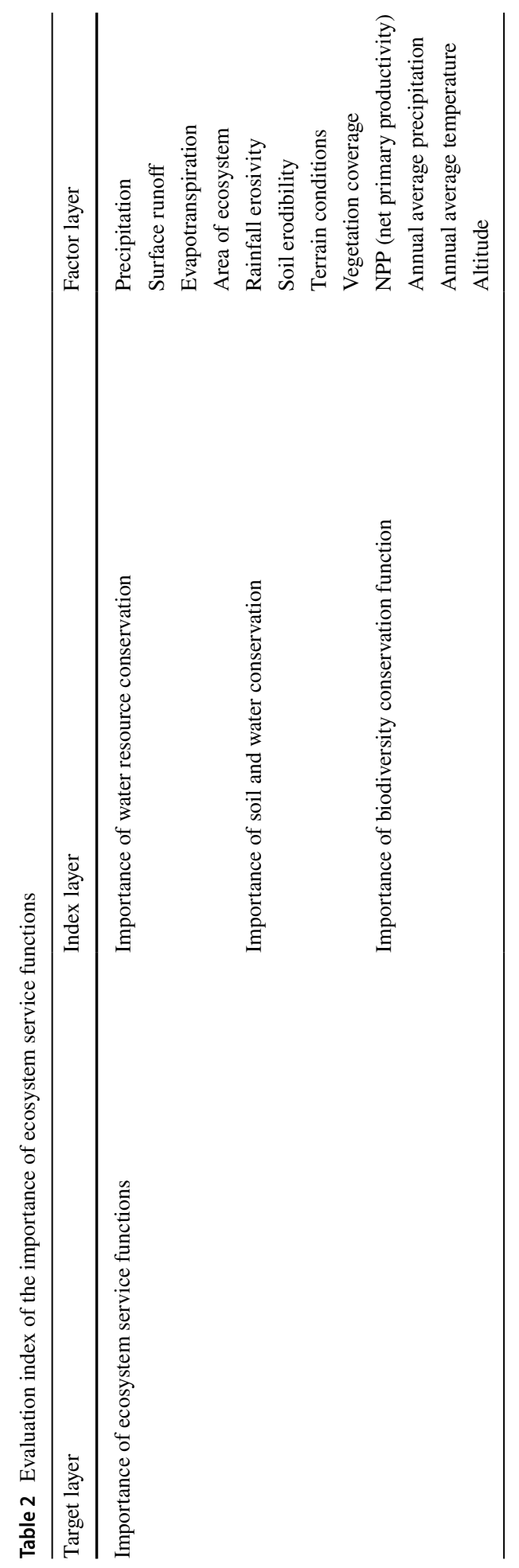


Table 3 The average surface runoff coefficient of each ecosystem type

\begin{tabular}{llc}
\hline Major ecosystem types & Subtypes of ecosystem & $\begin{array}{c}\text { The average surface } \\
\text { runoff coefficient }(\%)\end{array}$ \\
\hline Forest & Evergreen broad-leaved forests & 2.67 \\
& Evergreen needle-leaved forests & 3.02 \\
& Mixed broadleaf-conifer forests & 2.29 \\
& Deciduous broad-leaved forests & 1.33 \\
& Deciduous coniferous forest & 0.88 \\
Bush wood & Sparse woods & 19.20 \\
& Evergreen broad-leaved thickets & 4.26 \\
& Deciduous broad-leaved thickets & 4.17 \\
& Coniferous thickets & 4.17 \\
Grassland & Sparse thickets & 19.20 \\
& Meadow & 8.20 \\
& Grasslands & 4.78 \\
Wetland & Underbrush & 9.37 \\
& Sparse grass & 18.27 \\
& Wetland & 0.00
\end{tabular}

Development" which was issued by the Ministry of Natural Resources of the People's Republic of China in 2019.

3.1.1.2 Importance of soil and water conservation The soil and water conservation aims at reducing soil erosion by the interception and absorption of rainfall through vegetation and their roots. Normally, researchers adopt the revised universal soil loss equation (RUSLE) for simulation calculations (Liu et al. 2017). It considers the quantification of rainfall erosivity factor, soil erodibility factor, terrain factor (slope and length) and vegetation coverage factor comprehensively. By estimating the difference between potential and actual soil erosion as soil and water conservation value, RUSLE further characterizes the importance of soil and water conservation in Fengxian County. The specific calculation formula is as follows:

$$
A=R \times K \times L \times S \times(1-V)
$$

where $A$ denotes the soil and water conservation $\left(\mathrm{t} / \mathrm{hm}^{2} \mathrm{a}\right) ; R$ is the rainfall erodibility factor (MJ $\mathrm{mm} / \mathrm{hm}^{2} \mathrm{~h} \mathrm{a}$ ); $K$ is the soil erodibility factor $\left(\mathrm{t} \mathrm{hm^{2 }} \mathrm{h} / \mathrm{hm}^{2} \mathrm{MJ} \mathrm{mm}\right) ; L$ and $S$ are the terrain factors among which $L$ is the length factor and $S$ is the slope factor; and $V$ is the vegetation coverage factor.

3.1.1.3 Importance of biodiversity conservation function There is a complex interrelationship between biodiversity and ecosystem service functions, and the loss of biodiversity will greatly weaken the ecosystem's service functions (Patricia et al. 2016). The biodiversity conservation function is mainly manifested in the production and maintenance of biodiversity, climate regulation, flood and drought mitigation by ecosystems (Fischer et al. 2006; Hector et al. 2001). It is conducive to promote human understanding of the importance of ecosystem service functions by defining biodiversity conservation function. We chose four major influencing factors net primary productivity (NPP), annual average precipitation, annual average temperature and 
altitude, so as to evaluate the importance of biodiversity conservation function in Fengxian County. The calculation formula is:

$$
\mathrm{IBCF}=\mathrm{MNPP} \times F_{\text {pre }} \times F_{\text {tmp }} \times\left(1-F_{\text {alt }}\right)
$$

where IBCF denotes the importance of biodiversity conservation function; MNPP is the mean value of net primary productivity; and $F_{\text {pre }}, F_{\text {tmp }}$ and $F_{\text {alt }}$ represent the annual average precipitation, annual average temperature and altitude, respectively. They are all normalized to $0-1$ according to the extremum standardization method. At the same time, according to the topographical features of Fengxian County, we adopted the "species habitat factors" (Table 4) to modify the results.

\subsubsection{Ecological sensitivity}

Ecological sensitivity refers to the sensitivity of ecosystems to various environmental variability and human activities, and it can be used to reflect the possibility of ecological imbalances and ecological problems formation ( $\mathrm{Li}$ et al. 2014), so as to make sure the most sensitive and protective areas. By choosing the three factors soil and water losses sensitivity, desertification sensitivity in plain areas and salinization sensitivity as evidence for ecological sensitivity evaluation of Fengxian County, we divided the index layers' results (Table 5) into five levels as extremely sensitive, more sensitive, moderate sensitive, mild sensitive and non-sensitive, and their corresponding scores are 9, 7, 5, 3 and 1, respectively. Finally, we obtained the ecological sensitivity score by arithmetic average of the above three index layers' results. According to the specific scores and intergroup differences, we defined the five levels of ecological sensitivity based on the score thresholds of $>8,6-8,4-6,2-4$ and $<2$.

3.1.2.1 Soil and water losses sensitivity On the base of the universal soil loss equation (USLE), combining with the former scholars' related researches (Peng and Zhou 2019; Svoray and BenSaid 2009) and actual characteristics of Fengxian County, we identified the four factors rainfall erosivity, soil erodibility, relief amplitude and vegetation coverage as soil and water losses sensitivity indicators. The calculation formula is as follows:

$$
\mathrm{SS}=\sqrt[4]{R \times K \times \mathrm{LS} \times C}
$$

where SS is soil and water loss sensitivity; $R$ is the rainfall erodibility factor; $K$ is the soil erodibility factor; LS is the relief amplitude factor; and $C$ is the vegetation coverage factor.

3.1.2.2 Desertification sensitivity in plain areas The desertification sensitivity in plain areas is mainly characterized by the dry climate, low vegetation coverage and scattered surface soil structure which is vulnerable to wind erosion. According to the requirements of Provisional Technical Regulations of Ecological Function Zoning issued by the Ministry of Ecology and Environment of the People's Republic of China, we selected four evaluation indicators aridity index, sandy days over $5 \mathrm{~m} / \mathrm{s}$ in winter and spring, soil texture and vegetation coverage combining with the actual characteristics of Fengxian County. The calculation formula is (Liu et al. 2015):

$$
D=\sqrt[4]{I \times W \times K \times C}
$$




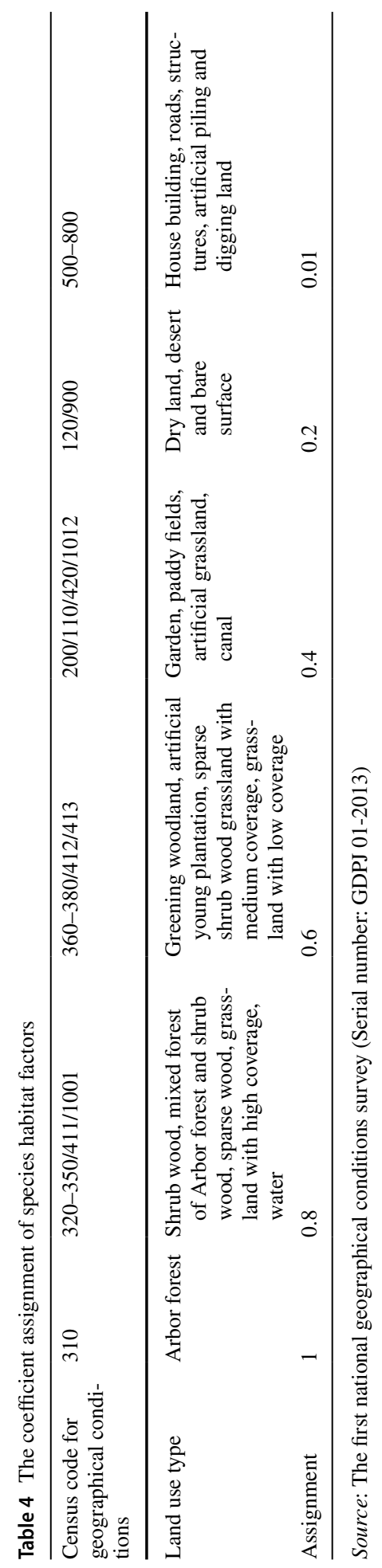


Table 5 Ecological sensitivity evaluation index

\begin{tabular}{lll}
\hline Target layer & Index layer & Factor layer \\
\hline Ecological sensitivity & Soil and water losses sensitivity & Rainfall erosivity \\
& Soil erodibility & Relief amplitude \\
& Vegetation coverage \\
& & Aridity index \\
& Sandy days over 5 m/s in winter and \\
& spring \\
& Soil texture \\
& Vegetation coverage \\
& Ratio of evaporation to precipitation \\
& Groundwater mineralization \\
& Groundwater depth \\
& Soil texture \\
\hline
\end{tabular}

where $D$ is the desertification sensitivity in plain areas; $I$ is the aridity index; $W$ is the sandy days over $5 \mathrm{~m} / \mathrm{s}$ in winter and spring; $K$ is the soil texture; and $C$ is the vegetation coverage factor.

3.1.2.3 Salinization sensitivity Salinization is mainly caused by drought climate, poor drainage, excessive groundwater level, unreasonable irrigation, etc. (Ouyang et al. 2000). According to the connotation of salinization and the actual situation of Fengxian County, we selected four factors including ratio of evaporation to precipitation, groundwater mineralization, groundwater depth and soil texture to evaluate salinization sensitivity. The specific calculation formula is:

$$
S=\sqrt[4]{I \times M \times D \times K}
$$

where $S$ is the salinization sensitivity; $I$ is the ratio of evaporation to precipitation; $M$ is the groundwater mineralization; $D$ is the groundwater depth; and $K$ is the soil texture.

\subsection{Ecological corridors identification}

The minimum cumulative resistance (MCR) model is calculated based on the GIS platform. It simulates the path of minimum cumulative resistance according to the cost of species from the ecological source to the destination, thereby constructing an ecological network. Currently, it has been widely used in the field of ecological network and ecological security structure construction (Zhang et al. 2017b; Chen et al. 2019). The identification of ecological corridors is based on the MCR model (Knaapen et al. 1992), and the formula is as follows:

$$
\mathrm{MCR}=f\left(\min \sum_{j=n}^{i=m} D_{i j} \times R_{i}\right)
$$


where MCR is the minimum cumulative resistance value; $D_{i j}$ is the spatial distance from source $j$ to landscape unit $i ; R_{i}$ is the resistance coefficient of landscape unit $i$ to species diffusion.

The resistance coefficient refers to cumulative resistance overcome by species as they travel through the heterogeneous landscapes. According to the assignments of various landscape types (Gurrutxaga et al. 2011; Kong et al. 2010) and actual situation of Fengxian County, the ecological resistance coefficients of forest land, grassland, garden land, cultivated land, unused land, water area and construction land were set to 1 , 10, 20, 30, 50, 300 and 500, respectively. Meanwhile, we used nighttime lighting data to modify the ecological resistance coefficients assigned by landscape types (Zhang et al. 2017b), so as to better embody the differences of ecological resistance coefficients among different spatial units. Finally, we obtained the ecological resistance surface. The calculation formula is:

$$
R_{i}=\frac{\mathrm{NL}_{i}}{\mathrm{NL}_{a}} \times R
$$

where $\mathrm{NL}_{i}$ is the nighttime lighting index of pixel $i$, $\mathrm{NL}_{a}$ is the mean nighttime lighting index of landscape type a to which the pixel $i$ belongs; and $R$ is the modified resistance coefficient assignments based on various landscape types.

\subsection{Ecological security pattern construction}

The construction of ecological security pattern based on the identification of "sources-corridors" essentially includes two core steps of ecological sources determination and ecological corridors extraction. Among these, the identification of ecological sources is based on ecological importance evaluation. Considering the actual situation of Fengxian County and the area of ecological sources comprehensively, we selected a certain area of patches with higher ecosystem importance as ecological sources. In addition, the extracted corridors among ecological sources are based on the MCR model. By using the spatial analysis tool in the GIS platform to calculate the minimum cost path, we constructed an ecological security pattern of Fengxian County which is consistent with its actual development.
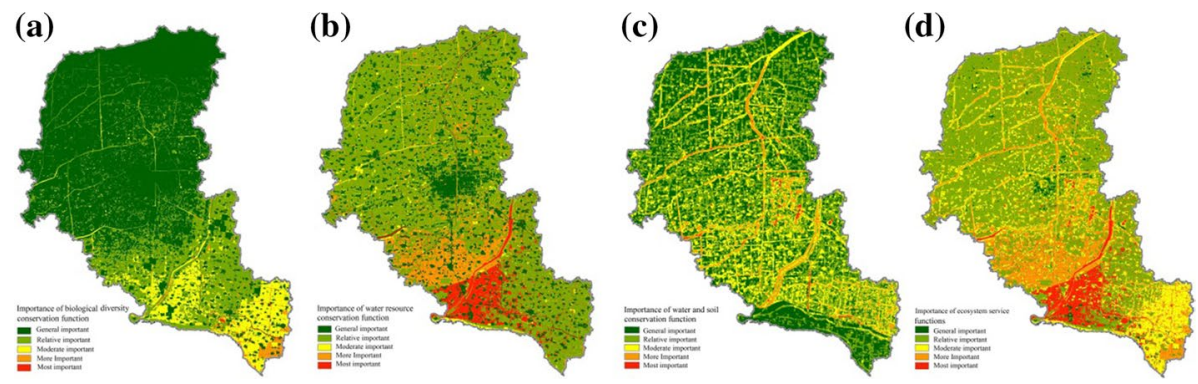

Fig. 3 Spatial differentiation of ecosystem service functions importance in Fengxian County 


\section{Results}

\subsection{Ecological importance evaluation}

The ecosystem service functions and their comprehensive evaluation results are shown as follows. In detail, the biodiversity conservation function score in the south area is higher than in the northern part (Fig. 3a). Moreover, the most important and more important areas are mainly distributed in Fanlou Town which is located in the south of the county. Relative important areas are mainly concentrated in the northern and central area which accounts for $67.82 \%$ of the whole county. The water resource conservation areas with high value are concentrated in the Dasha River, Dasha River Wetland and Dasha River Special Species Protection Area, while the low-value areas are mainly concentrated in the central urban area and the center of townships (Fig. 3b). Relative important water resource conservation areas account for $56.82 \%$ of the whole county. The soil and water conservation areas with high value are concentrated in the region nearby Dasha River and Fuxing River (Fig. 3c), while the low-value areas are concentrated in the northern and southern areas, and the distribution of other grades is relatively scattered. The whole county is dominated by soil and water conservation areas with relative importance and general importance, which accounts for $40.02 \%$ and $35.53 \%$, respectively.

We divided the evaluation results of ecosystem service function importance into five levels including general important, relative important, moderate important, more important and most important according to natural breaks method (Table 6). In terms of spatial distribution, more important and most important areas are mainly concentrated in the Dasha River, Dasha River Special Species Protection Area, Fuxing River and other rivers. Besides, moderate important areas are concentrated in Fanlou Town which is located in the southern part of Fengxian County. And the spatial distribution range of relative important areas is wider. General important areas are mainly concentrated in the urban center and administrative center areas of each township. In general, the importance degree of ecosystem service functions is mainly relative important, which accounts for $54.27 \%$ of the total county. What follows are moderate important and more important areas with the proportion up to $19.21 \%$ and $16.51 \%$, respectively, while the scale proportion of general important areas is the smallest.

\subsection{Ecological sensitivity evaluation}

The soil and water losses sensitivity in Fengxian County is mainly non-sensitive and mild sensitive which account for nearly $61 \%$ of the study area (Fig. $4 a$ ), while the proportion of the extremely sensitive area is only $0.03 \%$. The distribution differentiation of soil and water losses sensitivity results from the spatial differences among precipitation, soil and vegetation. In general, the soil and water losses sensitivity in the central and southeastern areas is relative high, and the northeastern area is at medium level. Moreover, the desertification sensitivity in plain areas of Fengxian County is dominated by non-sensitive and mild sensitive, which accounts for $37.01 \%$ and $23.56 \%$ of the whole area, respectively (Fig. 4b). On the whole, the heterogeneity of spatial distribution is very obvious. It demonstrates a medium level in the southwestern and northwestern regions. And the central region is extremely sensitive to desertification due to low vegetation coverage and high aridity index. The southeastern area presents a significant desertification sensitivity resulting from the 


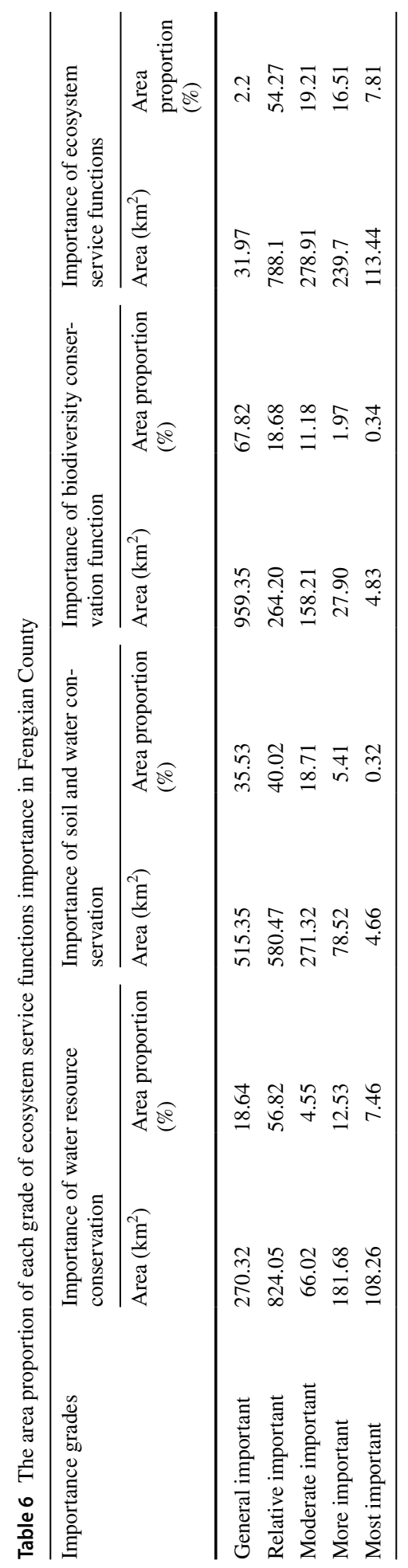



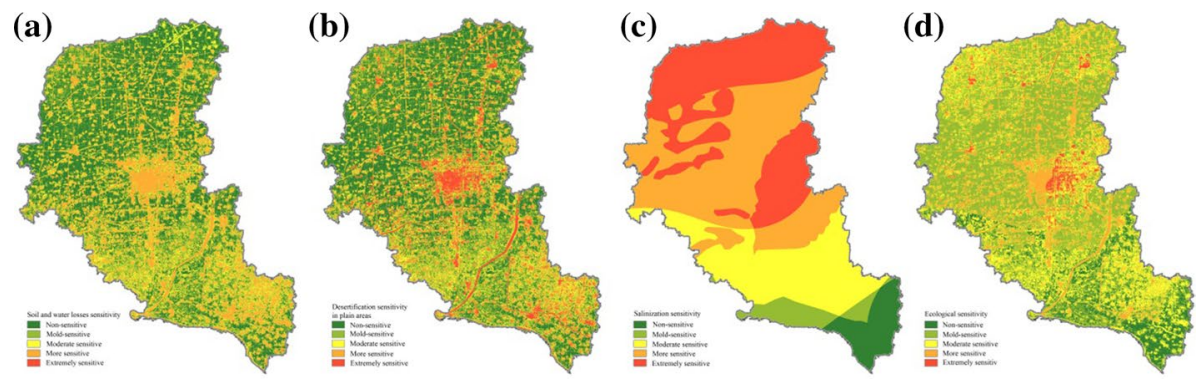

Fig. 4 Spatial differentiation of ecological sensitivity in Fengxian County

comprehensive effect of soil erodibility factor, aridity index and vegetation coverage factor. The salinization sensitivity of Fengxian County is mainly extremely sensitive, more sensitive and moderate sensitive, which accounts for $89.26 \%$ of the total area (Fig. 4c). From the aspect of spatial distribution, the northern region is extremely sensitive to salinization, while the central region is more sensitive. By contrast, the southern region is less salinized.

The spatial distribution of ecological sensitivity is basically consistent with single-factor sensitivity, which all present a higher sensitivity in the central region and lower sensitivity in the southern area. Areas with high sensitivity are mainly distributed in urban centers and water land. Moreover, moderate-sensitive areas are mainly distributed in the southeastern, southwestern and northern regions, while the central and northern regions present lower ecological sensitivity. On the whole, $46.98 \%$ of the whole county is mild-sensitive areas followed by moderate-sensitive areas. However, the size of extremely sensitive areas is only $35.53 \mathrm{~km}^{2}$ with the proportion up to $2.45 \%$ of the whole county (Table 7 ).

\subsection{Ecological sources identification based on ecological importance evaluation}

We obtained the results of the ecological importance of Fengxian County based on evaluation results of ecological service functions importance and ecological sensitivity according to the "maximum method." Next, we divided it into the minimum, lower, medium, higher and highest levels. The calculation results show that the area of ecological importance at the highest level is $148.54 \mathrm{~km}^{2}$, which accounts for $10.24 \%$ of the whole county. They are mainly distributed in the Dasha River and other areas with important water resource conservation function, as well as ecological sensitive areas such as administrative centers (Fig. 5). Moreover, the area of ecological importance at the higher level is $392.57 \mathrm{~km}^{2}$, which accounts for $27.07 \%$ of the whole county. They are approximately distributed in the Dasha River Special Species Protection Area, the underground drinking water source area in the central district and the Fuxing River. The areas of ecological importance at the medium level all mainly concentrated in the southeastern county. The areas of ecological importance at the lower level are widely distributed in the northern, central and southern county, among which the northern area shows a more centralized distribution, while the area of the minimum level is $0.77 \mathrm{~km}^{2}$ which only accounts for $0.05 \%$ of the whole county.

According to the evaluation results of ecological importance, we selected the patches at the higher and highest level as the ecological sources candidate areas in Fengxian County. It is noteworthy that there are many isolated and broken patches. Consider that only with a certain scale ecological sources can isolate external interference to the core 


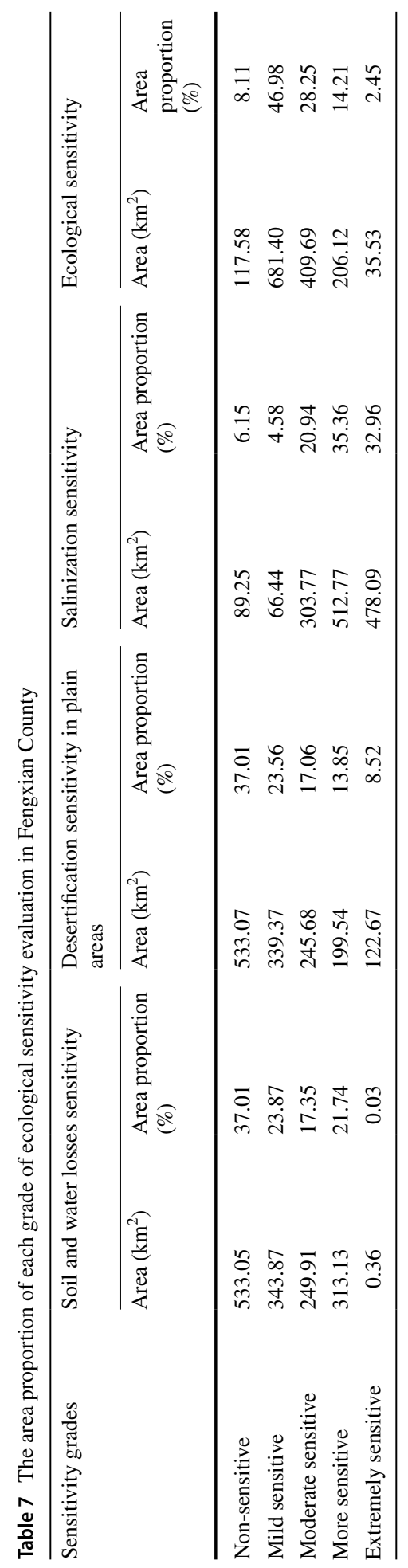




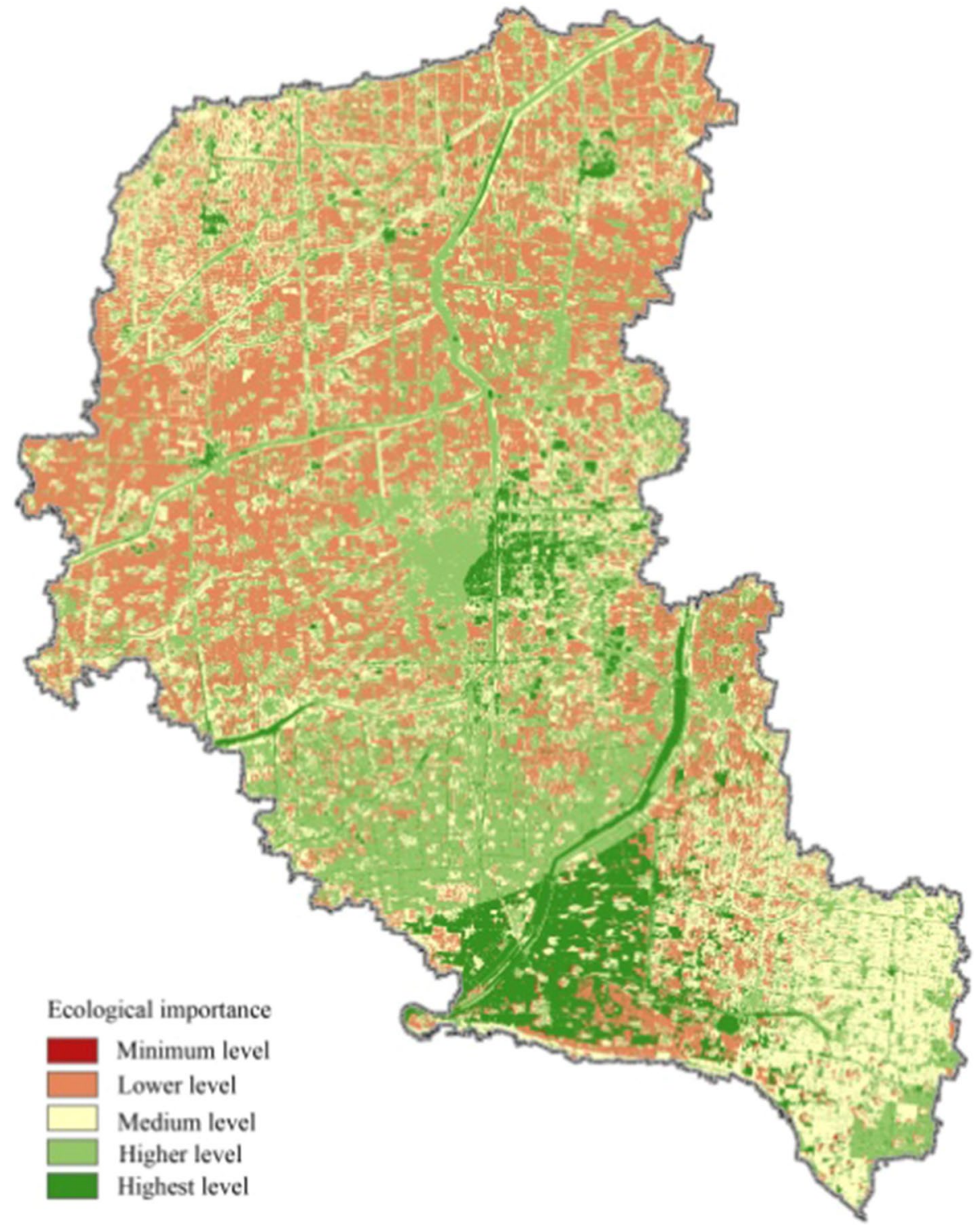

Fig. 5 Spatial differentiation of ecological importance in Fengxian County

area and play an important ecological role (Hao et al. 2019). Therefore, we selected the patches with the size of more than $10 \mathrm{~km}^{2}$ as ecological resources combined with the actual situation of Fengxian County. The final results show that the area proportion of ecological sources is $13.76 \%$, which is slightly smaller than the ecological red line area delineated by the current Ecological Redline Regional Protection Plan, but the spatial distribution is generally consistent with the ecological red line district. 


\subsection{Ecological corridors identification and ecological security pattern construction}

Ecological corridors are important channels connecting the ecological sources, as well as important carries of material and energy flow within the region. More importantly, they are key ecological components for maintaining ecological flow, ecological processes and ecological functions in connection with the region ( $\mathrm{Li}$ et al. 2004). Based on ecological sources and resistance surfaces, we used cost distance and cost path tools, as well as

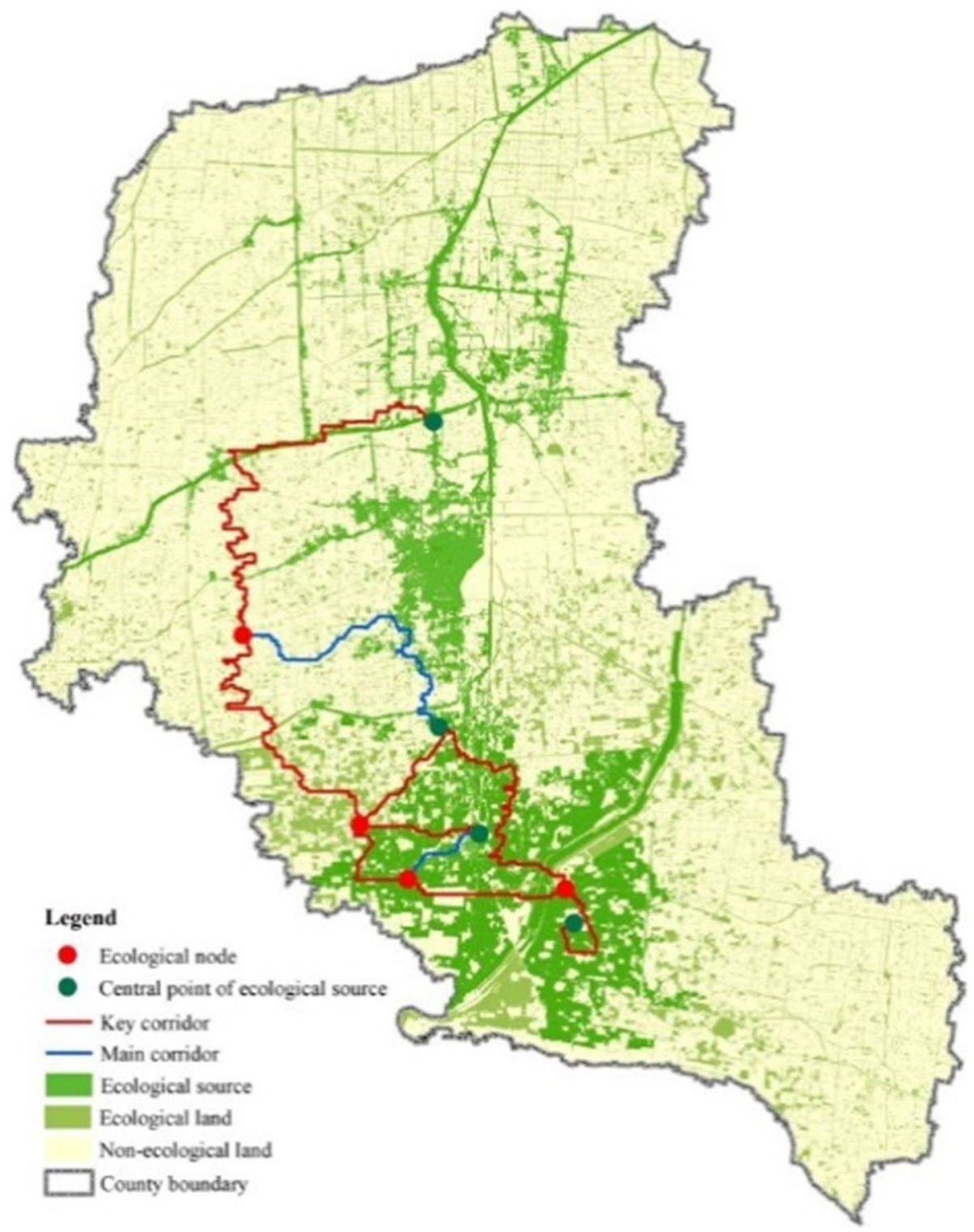

Fig. 6 Ecological security pattern construction in Fengxian County 
MCR model to construct the minimum cost paths and the secondary cost paths. In order to achieve the continuation of ecological functions and the connection of landscape patches at the regional scale, we defined the corridors with wide coverage and critical function in the minimum cost paths as key corridors combining with the ecological environment status and economic development needs of Fengxian County. Meanwhile, we regarded the corridors which play an important role in the connection of landscapes with smaller coverage in the secondary cost paths as main corridors (Fig. 6). According to statistical analysis, there are five key corridors and two main corridors with a total length of about $115.71 \mathrm{~km}$ and $26.22 \mathrm{~km}$, respectively, in Fengxian County. The ecological corridors are mainly distributed in the western and southwestern parts of Fengxian County with high vegetation coverage, which happens to be far away from the areas with strong humanity disturbances such as central urban area and township centers.

Urban expansion and intense human activities have changed the structure and functions of surface landscapes rapidly and strongly, leading to an increasingly intensified contradiction between economic development and ecological protection. The construction of regional ecological security pattern can protect important ecological patches effectively and coordinate the spatial conflicts between economic development and ecological protection. In general, it is one of the effective ways to construct land ecological barriers (Peng et al. 2017). The ecological security pattern of Fengxian County is composed of ecological sources, key corridors and main corridors (Fig. 6). Specifically, the ecological sources are mainly distributed in Dashahe Town, Songlou Town and Zhongyangli Street. It can provide important ecological protection for water resource conservation and biodiversity conservation in Dashahe Town and Songlou Town as concentrated ecological land distribution areas. Although Zhongyangli Street is the central urban area of Fengxian County which is mainly distributed by construction land, the construction of ecological sources in this area is no less important than other ecological sources as it is the distribution area of underground drinking water source in Fengxian County. Otherwise, the ecological corridors which connect ecological sources are distributed in the western and southwestern regions with a network form. And the direction of spatial paths is mainly extended from northwest to southeast.

The ecological security pattern of Fengxian County covers parallel layers and extends in branches, which shows a spatial pattern of "two vertical axes and five horizontal axes" as a whole. A complete complex system is formed through organic connections among ecological sources and ecological corridors. "One vertical axis" is located in the western county which extends from Zhaozhuang Town to Songlou Town. It achieves the continuity of ecological functions and the coherence of ecological processes in the western region to some extent. Moreover, "another vertical axis" is located in the central axis of Fengxian County, which connects the adjacent regions of underground drinking water source in Zhongyangli Street and the Dasha River water resource conservation area. It provides important support for water supply in the central core area. As for "five horizontal axes", apart from the key corridors extending from Zhaozhuang Town in the central part to Changdian Town in the northeastern area, a key ecological corridor is formed from the southern part of Songlou Town connecting eastward to the northern part of Dashahe Town. Two key corridors are also formed in the east-west direction and northeast-southwest direction within Songlou Town. In addition, a main ecological corridor in the northeast-southwest direction is formed in the southeastern part of Songlou Town. Furthermore, the central part of Wanggou Town extends eastward to Sunlou Street and leads to a main ecological corridor. All of them provide a good guarantee for the biodiversity and overall connectivity of landscape networks in Fengxian County. 


\subsection{Governance strategies of ecological security barriers and functional zoning}

According to the characteristics of ecological security pattern and regional functional zoning of Fengxian County, we constructed ecological security barriers including five axes, which are concentrated in the two regions of the central valuable agricultural eco-economic functional zone and southwestern forest and fruit eco-economic functional zone. We should improve the soil's ecological environment through the rational input of organic fertilizer in the central ecological zone. It is necessary to form a multi-dimensional planting mode while developing garden culture vigorously. On the other hand, we should also take advantage of deep sandy soil and abundant light in the western ecological zone. Apart from concentrating on the development of economic forest and fruit production as well as management activities, we did better continue to strengthen the awareness of ecological environmental protection. The ecological security barriers of Fengxian County are far from the main axis of urban development owing to the inevitable interference of human activities to the ecosystem, which is consistent with the objective law. For areas that are relatively consistent with the auxiliary axis of urban development, we should limit and regulate the responsible subjects of ecological security barriers. Through the participation and suggestions of stakeholders, as well as the implementation of relevant laws and regulations, relevant departments attempt to develop a negative list of environmental access for ecological security barriers to regulate human activities. As for the eco-tourism development axis which locates in the western part of Fengxian County, it is needed to explore the expansion model of the ecological network service path. In fact, as a self-organizing system with mutual feedback properties between functions and processes, it is conducive to the formation of different leisure experiences and the enhancement of well-being through appropriate green space opening policy. In addition, the northern ecological water system axis and the southern agricultural matrix axis are based on the conditions of groundwater, rivers and agricultural land. We should emphasize the coordination between natural background conditions and the structure of interest subjects, as well as the interest separation of ecological space. Finally, it is essential to establish a balanced development mechanism of ecological space that combines democratic rights with economic interests, avoiding the tragedy of commons (Fig. 7).

\section{Discussion and conclusions}

\subsection{Discussion}

The core research content of landscape ecology is to explore the interaction between landscape patterns and ecological processes. It will provide important spatial approaches for the construction of the ecological security pattern (Yu 1996). The ecological security pattern essentially refers to a potential ecological system spatial pattern among the landscape (Peng et al. 2017). By constructing an ecological security pattern, it is possible to effectively regulate ecological processes and ultimately achieve regional ecological security (Chen and Zhou 2005). The identification of ecological sources and ecological corridors plays a vital role in the construction of ecological security patterns. In this paper, the identification of the ecological sources has played an important role in constructing the ecological security pattern. Based on the ecological importance to identify ecological sources, while taking into account the quantitative 


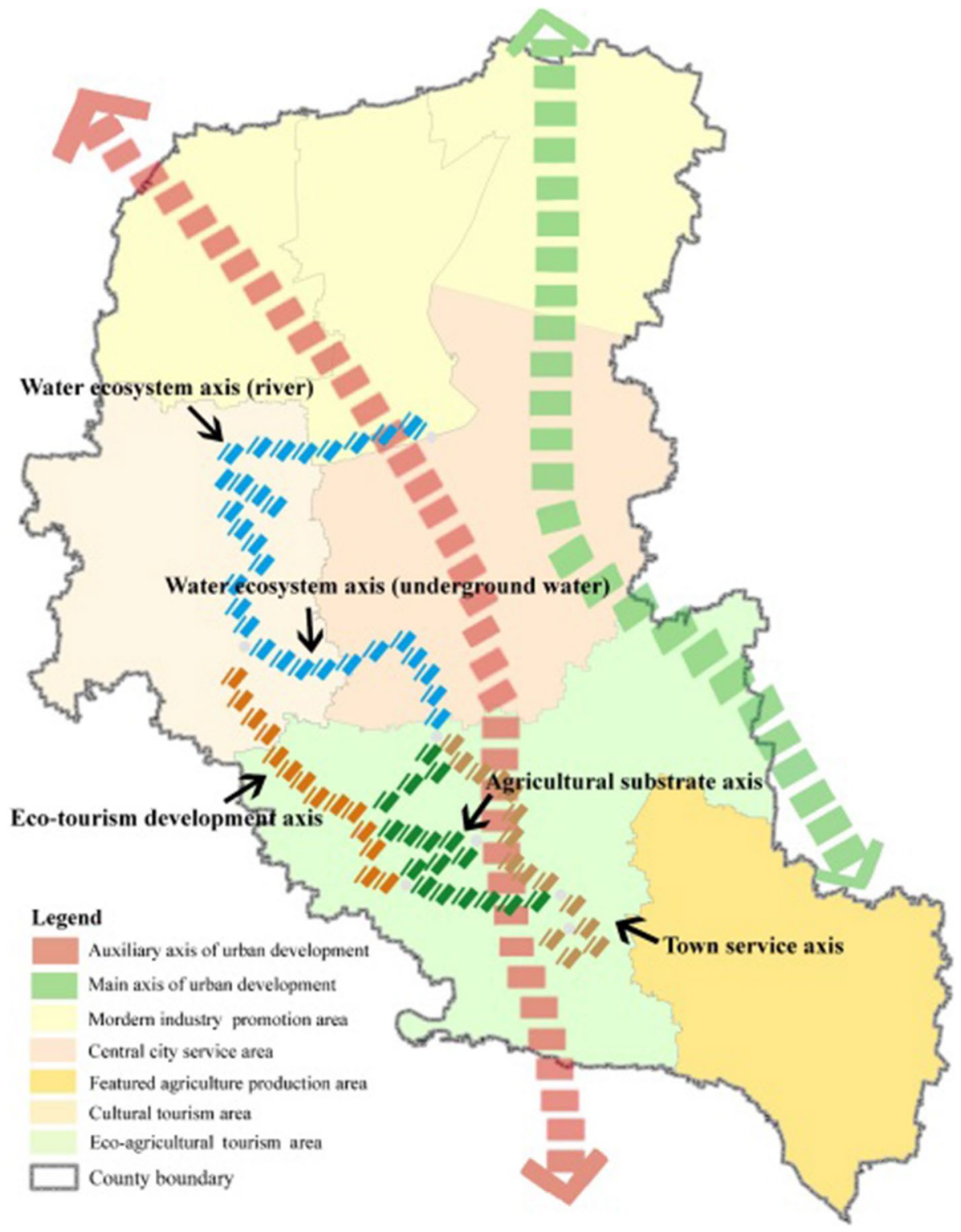

Fig. 7 Ecological security barriers and functional zoning in Fengxian County

value of ecosystem service functions and ecosystem sensitivity, the integration of ecological pattern quantification and ecological process evolution evaluation has been realized comprehensively to some extent (Guo et al. 2007). In this study, the identification of ecological sources has played an important role in constructing the ecological security pattern. In addition, the current methods and standards for the construction of ecological resistance surfaces have not been unified. Some studies have constructed resistance coefficients based on the perspective of biodiversity conservation (Balvanera 
et al. 2006; Zhang et al. 2017a). Some scholars also assigned resistance coefficients to grids units based on ecological sensitivity evaluation results (Fu et al. 2019). However, these methods do not take into account the versatility of landscape and the carrying role of multiple ecological processes. The construction of the resistance surface does not fully consider the regional differences, and it cannot reflect the interaction between the landscape pattern and the ecological process. To some extent, it will ultimately affect the authenticity of the simulation results. Therefore, this study obtained the ecological function resistance value based on various landscape types assignments. Meanwhile, considering the heterogeneity within the same landscape type and the impact of human activity intensity on ecological processes especially the regional migration of species, we adopted the night lighting data to modify the ecological function resistance value. Finally, we got the ecological resistance surface. On this basis, the ecological corridors are identified combining with the MCR model, thereby comprehensively constructing the ecological security pattern of Fengxian County. The ecological corridor identified by this method is basically within the ecological control line of Fengxian County. At the same time, it is confirmed that the identification method of the ecological sources and ecological corridors in this study is relatively reasonable. The recognition results are in good accordance with the actual situation of Fengxian County.

The ecological security pattern of Fengxian County is composed of ecological sources, key corridors and main corridors. Among which, the ecological sources play an important ecological protection role in water resource conservation and biodiversity conservation of Fengxian County. Meanwhile, the ecological sources are connected by ecological corridors which ensure the circulation and transition of materials and energy among ecological sources. In general, the existence of ecological corridors guarantees the continuity of ecological functions and the connectivity among the landscapes at the regional scale. It has a certain research significance and application value in constructing ecological security patterns and providing governance strategies of ecological security barriers at the small-scale county level. To some extent, it can offer a reference for the contradiction coordination between ecological environment and economic development in the developing countries and plain areas. Based on the construction of the ecological security pattern, this paper proposed ecological space optimization strategies in Fengxian County. It can provide a reference for alleviating the contradiction between economic development and ecosystem protection of small-scale county units in less developed regions. It also has important significance for optimizing the territory development pattern and promoting ecological civilization construction.

However, we only selected the importance of ecosystem service functions and ecological sensitivity to identify ecological sources comprehensively owing to the limitation of data acquisition and model accuracy. In future research, we will further explore more specific ecological security pattern protection methods based on detailed geospatial data. On the other hand, in view of the spatial mobility of material and energy, ecological security is simultaneously affected by ecosystems and ecological elements within and outside the region. Therefore, it may lead to negligence of ecological elements in the surrounding areas of Fengxian County due to the administrative boundary division. In the future, we will carry out researches on the construction of the ecological security pattern of Fengxian County under broader ecological background conditions. In addition, although we have considered the internal heterogeneity of landscape types when constructing ecological resistance surfaces, there are still further improvements in resistance coefficient assignments. 


\subsection{Conclusions}

The construction of ecological security pattern plays an important role in coordinating spatial conflicts between economic development and ecological protection. This study identified ecological sources based on the importance of ecosystem service functions and ecological sensitivity. Using landscape types assignments modified by nighttime light data and MCR model, we identified ecological corridors and finally constructed the ecological security pattern of Fengxian County. Based on these, we revealed the spatial structure characteristics of ecological security pattern so as to control and improve ecological problems from the source effectively, optimize the development pattern of territory space and promote the construction of ecological civilization. Our results show that: (1) the ecological sources of Fengxian County are mainly located in the central and southwestern part with the area of $199.58 \mathrm{~km}^{2}$ which accounts for $13.76 \%$ of the whole county. Moreover, the spatial distribution of ecological sources is highly coincident with the spatial location of the Dasha River, Fuxing River and the underground drinking water source area. (2) The ecological corridors of Feng County include two major categories of key corridors and main corridors with length of approximately $115.71 \mathrm{~km}$ and $26.22 \mathrm{~km}$, respectively. In addition, the spatial distribution of these corridors beyond the built-up areas with strong human disturbance as a whole, and mainly concentrate in the western and southwestern part of the county. (3) The ecological security network of Fengxian County is composed of four ecological sources and seven ecological corridors. These ecological components interact with each other to form a branched radiation ecological network space structure. (4) Based on the ecological security pattern and regional functional zoning characteristics of Fengxian County, we finally constructed the ecological security barrier including five axes. Furthermore, we explored the corresponding ecological space governance measures to optimize the ecological network of Fengxian County.

Acknowledgements This work was supported by the National Natural Science Foundation of China (Nos. 41430635 and 41901205).

Open Access This article is licensed under a Creative Commons Attribution 4.0 International License, which permits use, sharing, adaptation, distribution and reproduction in any medium or format, as long as you give appropriate credit to the original author(s) and the source, provide a link to the Creative Commons licence, and indicate if changes were made. The images or other third party material in this article are included in the article's Creative Commons licence, unless indicated otherwise in a credit line to the material. If material is not included in the article's Creative Commons licence and your intended use is not permitted by statutory regulation or exceeds the permitted use, you will need to obtain permission directly from the copyright holder. To view a copy of this licence, visit http://creativecommons.org/licenses/by/4.0/.

\section{References}

Balvanera, P., Pfisterer, A. B., Buchmann, N., He, J., Nakashizuka, T., Raffaelli, D., et al. (2006). Quantifying the evidence for biodiversity effects on ecosystem functioning and services. Ecology Letters, 9, 1146-1156.

Barral, M. P., \& Maceira, N. O. (2012). Land-use planning based on ecosystem service assessment: A case study in the Southeast Pampas of Argentina. Agriculture, Ecosystems \& Environment, 154(7), 34-43.

Carreño, L., Frank, F. C., \& Viglizzo, E. F. (2012). Tradeoffs between economic and ecosystem services in Argentina during 50 years of land-use change. Agriculture, Ecosystems \& Environment, 154(5), 68-77. 
Chen, L., Fu, B., \& Zhao, W. (2008). Source-sink landscape theory and its ecological significance. Frontiers of Biology in China, 3(2), 131-136.

Chen, L., Jing, Y., \& Sun, R. (2018). Urban eco-security pattern construction: Targets, principles and basic framework. Acta Ecologica Sinica, 38(12), 4101-4108.

Chen, D., Lan, Z., \& Li, W. (2019). Construction of land ecological security in Guangdong Province from the perspective of ecological demand. Journal of Ecology and Rural Environment, 35(7), 826-835.

Chen, X., \& Zhou, Cheng. (2005). Review of the studies on ecological security. Progress in Geography, 24(6), 8-20. https://doi.org/10.3969/j.issn.1007-6301.2005.06.002.

Cheng, G., \& Shi, P. (2004). Benefits of forest water conservation and its economical value evaluation in upper reaches of Yangtse River. Science of Soil and Water Conservation, 4, 17-20.

Cumming, G. S., \& Allen, C. R. (2017). Protected areas as social-ecological systems: Perspective from resilience and social-ecolocial systems theory. Ecological Applications, 27(6), 1709-1717.

Daily, G. C. (1997). Nature's service: Social dependence on natural ecosystem. Washington D.C.: Island Press.

Elvidge, C., Sutton, P., Ghosh, T., Tuttle, B., Baugh, K., Bhaduri, B., et al. (2009). A global poverty map derived from satellite data. Computers \& Geosciences, 35, 1652-1660.

Fang, C., Cui, X., Li, G., Bao, C., Wang, Z., \& Ma, H. (2019). Modeling regional sustainable development scenarios using the Urbanization and Eco-environment Coupler: Case study of Beijing Tianjin-Hebei urban agglomeration, China. Science of the Total Environment, 689, 820-830.

Fischer, J., Lindenmayer, D., \& Manning, A. (2006). Biodiversity, ecosystem function, and resilience: Ten guiding principles for commodity production landscapes. Frontiers in Ecology and the Environment, 4(2), 80-86.

Frelichova, J., Vackar, D., Partl, A., Louvkova, B., Harmackova, Zuzana V., \& Lorencoa, E. (2014). Integrated assessment of ecosystem services in the Czech Republic. Ecosystem Services, 8, 110-117.

Fu, W., Liu, S., Degloria, S., Dong, S., \& Beazley, R. (2010). Characterizing the "fragmentation-barrier" effect of road networks on landscape connectivity: a case study in Xishuangbanna, Southwest China. Landscape and Urban Planning, 95(3), 122-129.

Fu, C., Xu, Y., Bundy, A., Gruss, A., Coll, M., Heymans, J., et al. (2019). Making ecological indicators management ready: Assessing the specificity, sensitivity, and threshold response of ecological indicators. Ecological Indicators, 105, 16-28.

Guo, R., Miao, C., Li, X., \& Chen, D. (2007). Eco-spatial structure of urban agglomeration. Chinese Geographical Science, 17(1), 28-33.

Gurrutxaga, M., Lozano, P., \& Barrio, G. (2010). GIS-based approach for incorporating the connectivity of ecological networks into regional planning. Journal for Nature Conservation, 18(4), 318-326.

Gurrutxaga, M., Rubio, L., \& Saura, S. (2011). Key connectors in protected forest area networks and the impact of highways: A transnational case study from the Cantabrian Range to the Western Alps (SW Europe). Landsc Urban Plan, 101, 310-320.

Hao, Y., Zhang, N., Du, Y., Wang, Y., Zheng, Y., \& Zhang, C. (2019). Construction of ecological security pattern based on habitat quality in Tang County, Hebei, China. The Journal of Applied Ecology, 30(3), 1015-1024.

Hector, A., Joshi, J., Lawler, S. P., Spehn, E. M., \& Wilby, A. (2001). Conservation implications of the link between biodiversity and ecosystem functioning. Oecologia, 129(4), 624-628.

Kareiva, P., Watts, S., Mcdonald, R., \& Boucher, T. (2007). Domesticated nature: Shaping landscapes and ecosystems for human welfare. Science, 316(5833), 1866-1869.

Klar, N., Herrmann, M., Henning-Hahn, M., Pott-Dorfer, B., Hofer, H., \& Kramer-Schadt, S. (2012). Between ecological theory and planning practice: (Re-) connecting forest patches for the wildcat in Lower Saxony, Germany. Landscape and Urban Planning, 105(4), 376-384.

Knaapen, J. P., Scheffer, M., \& Harms, B. (1992). Estimating habitat isolation in landscape planning. Landscape and Urban Planning, 23(1), 1-16.

Kong, F., Yin, H., Nakagoshi, N., \& Zong, Y. (2010). Urban green space network development for biodiversity conservation: Identification based on graph theory and gravity modeling. Landscape and Urban Planning, 95, 16-27.

Li, Y., Shi, Y., Qureshi, S., Bruns, A., \& Zhu, X. (2014). Applying the concept of spatial resilience to socio-ecological systems in the urban wetland interface. Ecological Indicators, 42, 135-146.

Li, J., Song, C., Cao, L., Zhu, F., Meng, X., \& Wu, J. (2011). Impacts of landscape structure on surface urban heat islands: A case study of Shanghai, China. Remote Sensing of Environment, 115(12), 3249-3263.

Li, W., Wang, Y., Peng, J., \& Li, G. (2004). Landscape spatial changes in Shenzhen and their driving factors. Chinese Journal of Applied Ecology, 8, 1403-1410. 
Li, S., Xiao, W., Zhao, Y., Xu, J., Da, H., \& Lv, X. (2019). Quantitative analysis of the ecological security pattern for regional sustainable development: Case study of Chaohu Basin in eastern China. Journal of Urban Planning and Development, 145(3), 04019009.

Liu, M. (2016). The ecological security pattern of China's energy consumption based on carbon footprint. Landscape Architecture Frontiers, 4(5), 10-16.

Liu, J., Gao, J., Ma, S., Wang, W., \& Zou, C. (2015). Evaluation of ecological sensitivity in China. Journal of Natural Resources, 30(10), 1607-1616.

Liu, S., Wang, D., Li, H., Li, W., Wu, W., \& Zhu, Y. (2017). The ecological security pattern and its constraint on urban expansion of a black soil farming area in Northeast China. Isprs International Journal of Geo-information, 6(9), 263.

Mellander, C., Lobo, J., Stolarick, K., \& Matheson, Z. (2015). Night-time light data: A good proxy measure for economic activity? PLoS ONE, 10(10), 0139799.

Ouyang, Z., Wang, X., \& Miao, H. (2000). China's eco-environmental sensitivity and its spatial heterogeneity. Acta Ecologica Sinica, 20(1), 9-12.

Patricia, P. L., Vazquez, L. B., Sarmiento-Aguilar, Rausel, Douterlungne, D., \& Valenzuela-Galvan, D. (2016). Influence of human activities on some medium and large-sized mammals' richness and abundance in the Lacandon Rainforest. Journal for Nature Conservation, 34, 75-81.

Peng, J., Yang, Y., Liu, Y., Hu, Y., Du, Y., \& Meersmans, J. (2018). Linking ecosystem services and circuit theory to identify ecological security patterns. Science of the Total Environment, 644, 781-790.

Peng, J., Zhao, H., Liu, Y., \& Wu, J. (2017). Research progress and prospect on regional ecological security pattern construction. Geographical Research, 36(3), 407-419.

Peng, W., \& Zhou, J. (2019). Development of land resources in transitional zones based on ecological security pattern: a case study in China. Natural Resources Research, 28(S1), S43-S60.

Plieninger, T., Kizos, T., Bieling, C., Dû-Blayo, L. L., Budniok, M. A., Bürgi, M., et al. (2015). Exploring ecosystem change and society through a landscape lens: Recent progress in European landscape research. Ecology and Society, 20(2), 60-62.

Scholte, Smmantha S. K., van Teeffelen, Astrid J. A., \& Verburg, Peter H. (2015). Integrating socio-cultural perspectives into ecosystem service valuation: A review of concepts and methods. Ecological Economics, 114, 67-78.

Song, L., \& Qin, M. (2016). Identification of ecological corridors and its importance by integrating circuits and found. Chinese Journal of Applied Ecology, 27(10), 3344-3352.

Svoray, T., \& Ben-Said, S. (2009). Soil loss, water ponding and sediment deposition variations as a consequence of rainfall intensity and land use: A multi-criteria analysis. Earth Surface Processes and Landforms, 35(2), 202-216.

Tang, M., Wu, C., Zhou, Z., Lord, E., \& Zheng, Z. (2011). Multipurpose greenway planning for changing cities: A framework integrating priorities and a least-cost path mode. Landscape and Urban Planning, 103(1), 1-14.

Wang, D., Chen, J., Zhang, L., Sun, Z., Wang, X., Zhang, X., et al. (2019). Establishing an ecological security pattern for urban agglomeration, taking ecosystem services and human interference factors into consideration. PeerJ, 7, e7306.

Wang, M., \& Xu, H. (2018). Temporal and spatial changes of urban impervious surface and its influence on urban ecological quality: A comparison between Shanghai and New York. Chinese Journal of Applied Ecology, 29(11), 3735-3746.

Wang, Y., Li, X., Zhang, Q., Li, J., \& Zhou, X. (2018). Projections of future land use changes: Multiple scenarios -based impacts analysis on ecosystem services for Wuhan city, China. Ecological Indicators, 94(1), 430-445.

Xin, X., Shao, L., Gu, C., \& Li, J. (2016). From "what to do" to "what not to do": the construction of county spatial control system based on "multiple planning coordination". Urban Development Studies, 23(3), $15-21$.

$\mathrm{Xu}, \mathrm{X}$. (2018). Spatial distribution data of soil texture in China. Registration and Publishing System of Chinese Academy of Sciences Resource and Environment Science Data Center. http://www.resdc.cn.

Yang, Z., Jiang, Z., Guo, C., Yang, X., Xu, X., Li, X., et al. (2018). Construction of ecological network using morphological spatial pattern analysis and minimal cumulative resistance models in Guangzhou City, China. Chinese Journal of Applied Ecology, 29(10), 3367-3376.

Yao, L., Li, X., Li, Q., \& Wang, J. (2019). Temporal and spatial changes in coupling and coordinating degree of new urbanization and ecological-environmental stress in China. Sustainability, 11(4), 1171.

$\mathrm{Yu}$, K. (1996). Security patterns and surface model in landscape ecological planning. Landscape and urban planning, 36(1), 1-17.

Yu, K. (1999). Landscape ecological security patterns in biological conservation. Acta Ecologica Sinica, 19(1), 8-15. 
Zhang, L., Peng, J., Liu, Y., \& Wu, J. (2017a). Coupling ecosystem services supply and human ecological demand to identify landscape ecological security pattern: a case study in Beijing-Tianjin-Hebei region, China. Urban Ecosystem, 20, 701-714.

Zhang, J., Qiao, Q., Liu, C., Wang, H., \& Pei, S. (2017b). Ecological land use planning for Beijing city based on the minimum cumulative resistance model. Acta Ecologica Sinica, 37(19), 6313-6321.

Publisher's Note Springer Nature remains neutral with regard to jurisdictional claims in published maps and institutional affiliations.

\section{Affiliations}

\section{Xingxing $\mathrm{Jin}^{1} \cdot$ Luyao Wei $^{1}$ (D) $\cdot$ Yi Wang $^{2} \cdot$ Yuqi $\mathrm{Lu}^{1,3,4}$}

Luyao Wei

lmyxpeking@163.com

$\triangle$ Yuqi Lu

luyuqi@263.net

Xingxing Jin

xxjin91@126.com

Yi Wang

wangyearn@163.com

1 School of Geographical Science, College of Geography Science, Nanjing Normal University, No. 1 Wenyuan Road, Nanjing 210023, Jiangsu, China

2 School of Economics and Management, Nanjing University of Science and Technology, Nanjing 210094, China

3 Jiangsu Center for Collaborative Innovation in Geographical Information Resource Development and Application, Nanjing 210023, Jiangsu, China

4 Key Laboratory of Virtual Geographic Environment Ministry of Education in Nanjing Normal University, Nanjing 210023, Jiangsu, China 